\title{
MiR-27b Impairs Adipocyte Differentiation of Human Adipose Tissue-Derived Mesenchymal Stem Cells by Targeting LPL
}

\author{
Xumin $\mathrm{Hu}^{\mathrm{a}}$ Jianhua Tang ${ }^{\mathrm{b}} \quad$ Xuyun Hu${ }^{\mathrm{c}}$ Peng Bao ${ }^{\mathrm{d}} \quad$ Jinxin Pan ${ }^{\mathrm{a}} \quad$ Zhipeng Chen $^{\mathrm{a}}$ \\ Jiaqi Xian \\ aDepartment of Orthopedics, Sun Yat-sen Memorial Hospital, Sun Yat-sen University, Guangzhou, \\ Guangdong, 'Department of Orthopedics, People's Hospital of Gaozhou, Gaozhou, Guangdong, China, \\ 'Department of Newborn Medicine, Boston Children's Hospital, Harvard Medical School, Boston, \\ MA, USA, 'Medical Department, Sun Yat-sen Memorial Hospital, Sun Yat-sen University, Guangzhou \\ Guangdong, China
}

\section{Key Words}

Adipose tissue - Adipogenic differentiation - MiR-27b - Mesenchymal stem cells (MSCs) • Metabolism $•$ LPL

\begin{abstract}
Background/Aims: In this study, the molecular mechanisms of miR-27b and lipoprotein lipase (LPL) that regulate human adipose-derived mesenchymal stem cells (hASCs) adipogenic differentiation were detected. Methods: Microarray analysis was applied to screen for differentially expressed miRNAs and mRNA during hASCs adipocyte differentiation induction. MiR-27b and $L P L$ were found to have abnormal expression. Then, a dual luciferase reporter assay was employed to validate the targeting relationship between miR-27b and $L P L$. We also utilized qRT-PCR, western blot, cellular immunofluorescence and an oil red $O$ staining assay to analyze the regulation of miR-27b and $L P L$ during adipogenic differentiation. Results: The microarray analysis demonstrated that, during adipogenic differentiation, miR-27b was downregulated, while $L P L$ was up-regulated but tended to become stable 14 days after induction. A dual luciferase reporter assay confirmed the negative targeting regulatory relationship between miR-27b and LPL. After overexpressing and silencing miR-27b, LPL was found to be reversely regulated by miR-27b according to $q R T-P C R$ and western blot. The fat-formationrelated biomarkers CCAAT-enhancer binding protein $\alpha$ (C/EBP $\alpha$ ) and peroxisome proliferatoractivated receptors $\gamma$ (PPAR $\gamma$ ) had decreasing levels after over-expressing miR-27b or knockdown of $L P L$ followed by adipogenic differentiation. Meanwhile, the oil red $\mathrm{O}$ staining assay revealed that the accumulation of lipid droplets decreased. There was no change in the expression of c/EBP $\alpha, P P A R y$, or lipid droplet accumulation when overexpressing miR-27b and LPL. Conclusion: During the adipogenic differentiation of hASCs, miR-27b expression decreased, and $L P L$ expression increased. The abnormal expression of miR-27b and $L P L$ effectively regulated the adipogenic differentiation of hASCs.
\end{abstract}

X. Hu and J. Tang contributed equally to this work. 


\section{Cellular Physiology Cell Physiol Biochem 2018;47:545-555 and Biochemistry Published online: May 24, $2018 \quad \begin{aligned} & \text { DOI: 10.1159/000489988 } 2018 \text { The Author(s). Published by S. Karger AG, Basel } \\ & \text { www.karger.com/cpb }\end{aligned}$}

Hu et al.: Up-Regulation of miR-27b Inhibits Adipogenic Differentiation of hASCs

\section{Introduction}

Adipose tissues, which may be brown or white, store lipids to maintain energy metabolism and serve as an endocrine organ. They are organized to form a large organ with a discrete anatomy, specific vascular and nerve supplies, complex cytology, and high physiological plasticity [1,2]. White adipose tissue is widely available and has the ability to differentiate into other tissue types. Monitoring the alteration of adipose-derived stem cells (ASCs) can provide a promising future in the field of tissue engineering and regenerative medicine [3]. First, ASCs are recruited by certain stimulation, following which they are transformed into preadipocytes and then ultimately turn into mature adipocytes [4]. During adipogenesis, several transcription factors such as peroxisome proliferator-activated receptor- $\gamma$ (PPAR $\gamma$ ) and members of the CCAAT/enhancer-binding family of proteins (c/EBP $\alpha$ ) participate in regulating adipogenesis $[5,6]$. ASCs not only possess the ability to differentiate into multiple tissue types but also have immune-modulatory properties, which are similar to those of mesenchymal stem cells (MSCs) [7]. Nevertheless, the regulatory mechanisms of the ASCselicited immune-modulatory activity by specific microRNA (miRNA) remain unexplored.

The small non-coding RNAs are abbreviated as microRNAs or miRNAs, which target the 3'-untranslated regions (UTRs) of their target mRNAs to regulate gene expression [8-10]. Numerous biological processes, including cell fate determination in embryonic stem cells as well as the regulation of adipogenic and osteogenic differentiation, have been confirmed to be regulated by miRNAs in human bone marrow mesenchymal stem cells (hBMSCs) [11]. Many studies have shown that miR-27 plays important roles in lipid metabolism and adipogenesis [12]. The miR-27 family has two isoforms, intergenic miRNA miR-27a and intronic miRNA miR-27b [13]. The sequences of miR-27a and miR-27b are highly conserved among different species. There are reports that miR-27a/b has a negative regulatory effect on peroxisome proliferator-activated receptor gamma (PPAR $\gamma$ ) and C/EBP alpha in 3T3-L1 cells and hASCs [14]. Moreover, miR-27b have been found to play important roles in the development and progression of neoplastic diseases by bonding to key targets. miR-27b can not only target MET to suppress proliferation and promote apoptosis in diffuse large B-cell lymphoma [15], but it can also target receptor tyrosine kinase like orphan receptor 1 (ROR1) to suppress cell proliferation in gastric cancer [16]. Additionally, miR-27b overexpression enhanced the docetaxel sensitivity of prostate cancer partly through inhibiting EMT by targeting ZEB1 [17]. Therefore, performing investigations into miR-27b is a promising direction for human health promotion. However, there is still a problem to be solved in that the specific roles of these miRNAs in pluripotent cell commitment to adipocyte differentiation are unclear.

Lipoprotein lipase $(L P L)$, most abundantly expressed in adipose tissue, macrophages, heart and skeletal muscle, acts as a gatekeeper for the entry of fatty acids into tissues and controls systemic lipid partitioning, which is essential for energy homeostasis of the body [18]. Human $L P L$ plays a significant role in regulating triglyceride (TG) levels by hydrolyzing TGs in TG-rich lipoproteins as the first step in their metabolism [19]. Furthermore, $L P L$ hydrolyzes the triglyceride-rich core of chylomicrons (CM) and very low density lipoproteins (VLDL) [20]. The molecular mechanism whereby $L P L$ regulates hASCs adipogenic differentiation and the interactive relationship between $L P L$ and miR-27b needs numerous studies.

This study aimed to provide a new perspective for obesity, diabetes mellitus, and other obesity related metabolic diseases by studying the effect of miRNA on adipogenic differentiation of human adipose-derived stem cells (hASCs) and exploring its molecular mechanism. We investigated the relationship between miR-27b and $L P L$ and their effects on adipogenic differentiation of hASCs.

\section{Materials and Methods}

Clinical specimens

Adipose tissue samples were collected from healthy female patients who were undergoing double eyelidplasty treatment at the People's Hospital of GaoZhou. The donors were between 20 and 30 years old. 


\section{Cellular Physiology Cell Physiol Biochem 2018;47:545-555 \begin{tabular}{l|l} 
and Biochemistry Published online: May 24, 2018 & $\begin{array}{l}\text { D) } 2018 \text { The Author(s). Published by S. Karger AG, Basel } \\
\text { www.karger.com/cpb }\end{array}$
\end{tabular} \\ Hu et al.: Up-Regulation of miR-27b Inhibits Adipogenic Differentiation of hASCs}

hASCs were extracted from the abovementioned adipose tissues and then treated with or without adipogenic induction for further study. All experiments were approved by the Ethics Committee of the People's Hospital of GaoZhou, and informed consent was obtained from all of the participating patients.

\section{Microarray analysis}

A miRNA microarray platform GPL16700 was used to analyze the expression profiles of miRNA in two adipogenic differentiated samples and two normal hASCs samples in GSE72429 (www.ncbi.nlm.nih. gov/geo/query/acc.cgi?acc=GSE72429). Differentially expressed miRNAs were identified by setting the fold change at 2 and the p-value at $P<0.05$. The GPL16686 platform was utilized to detecting the differentially expressed mRNAs of four samples in GSE77532. Multivariate analysis of variance (MANOVA) was performed to extract the overall characteristics, and ANOVA was performed to analyze the mRNA expression profile on the first day, the seventh day, the fourteenth day and the twenty-first day after adipogenic differentiation. Differentially expressed mRNAs were identified by setting $P<0.001$.

\section{Cell culture and isolation}

We successfully extracted the hASCs based on previously published methods [21]. Then, Dulbecco's modified Eagle's medium-low glucose (DMEM-LG, Invitrogen, Carlsbad, CA, USA) was employed to culture the hASCs cells at room temperature in a $5 \% \mathrm{CO}_{2}$ humidified atmosphere, containing $10 \%$ fetal bovine serum (FBS), $100 \mathrm{U} / \mathrm{ml}$ penicillin and $100 \mu \mathrm{g} / \mathrm{ml}$ streptomycin. The medium was replaced and non-adherent cells were removed after 24 hours. After primary culture for 7 days, subculture was performed at 1:3 ratios, and the third generation was used for further studies.

\section{Adipogenic induction of $h A S C S$}

The third-generation cells were selected for testing and cultured in 6-well plates at a density of $1 \times 10^{5}$ cells/well. Then, the medium was changed to high glucose adipogenic induction medium (H-DMEM) after the cells achieved a $90 \%$ concentration. The adipogenic induction was performed under the condition of 10 $\%$ FBS (Sigma-Aldrich, St. Louis, MO, USA), $1 \times 10^{-7} \mathrm{~mol} / \mathrm{L}$ dexamethasone (Sigma), $0.5 \mathrm{mmol} / \mathrm{L}$ 3-isobutyl-1methyl-xanthine (Sigma), and $5 \mu \mathrm{g} / \mathrm{mL}$ ascorbic acid (Sigma).

\section{Cell transfection}

The transfection experiments were performed using Lipofectamine ${ }^{\circledR} 2000$ (Invitrogen, Carlsbad, CA, USA) according to the manufacturer's instruction. The synthesized miR-27b mimics, control mimics, pcDNA3.1-LPL and pcDNA3.1-control were obtained from Invitrogen (Carlsbad, CA, USA). Santa Cruz Biotechnology (Santa Cruz, CA, USA) was employed to synthesize the miR-27b inhibitor, LPL shRNA and control shRNA. The recombinant plasmids were transformed into the hASCs and they were then amplified, and the positive clones were picked and sequenced. Logarithmic growth phase cells were taken and counted with a counting plate, then placed in culture plates at the appropriate density (approximately $50 \%$ ). The cells were incubated at $37^{\circ} \mathrm{C}$ for 24 hours until all of the cells were stretched out and evenly distributed.

\section{Oil red $O$ staining}

The cells were washed with the PBS buffer 2-3 times and fixed with $3.7 \%$ paraformaldehyde for $2 \mathrm{~min}$. Then, the cells were stained for 1 hour by incubating with oil red 0 after washing twice in distilled water. Using glycerin gelatin, the slides were sealed and we removed the excess water. Cell morphology and the staining patterns were then examined using a phase contrast microscope (Zeiss, Germany).

\section{QRT-PCR assay}

We collected the total RNA by using the TRIzol reagent (Invitrogen, Carlsbad, CA, USA) and the extracted RNAs were used to obtain reverse-transcribed cDNA following the manufacturer's instructions with a PrimeScript II 1st Strand cDNA Synthesis Kit (TaKaRa, Tokyo, Japan). Following the instructions of the SYBR Premix Ex Taq (TaKaRa, Tokyo, Japan), the obtained cDNAs were amplified. U6 and GAPDH were applied as the internal reference for detecting miR-27b and $L P L$ mRNA, respectively. The experiments were performed three times, and the $2^{-\Delta \Delta c t}$ method was used to quantify the relative expression levels of miR-27b and $L P L$. The primer sequences used are shown in Table 1. 


\section{Cellular Physiology Cell Physiol Biochem 2018;47:545-555 and Biochemistry Published online: May 24, $2018 \quad$\begin{tabular}{l|l} 
DOI: 10.1159/000489988 2018 The Author(s). Published by S. Karger AG, Basel \\
www.karger.com/cpb
\end{tabular} \\ Hu et al.: Up-Regulation of miR-27b Inhibits Adipogenic Differentiation of hASCs}

Western blot

The total proteins in hASCs were extracted and the protein concentrations were measured using a Bradford protein assay (Bio-Rad, Hercules, CA, USA). After inactivation, the samples were electrophoresed on SDS-PAGE and transferred to nitrocellulose membranes via an iBlot Dry Blotting Transfer System (Life Technologies Corp., Gaithersburg, MD, USA). A PBS buffer containing $5 \%$ non-fat milk and $0.1 \%$ Tween was applied and used to incubate the membranes for 2 hours, followed by anti-LPL (ab21356, 1: 1000, Abcam, MA, USA), anti-c/EBP $\alpha$ (ab32358, 1: 1000, Abcam), anti-PPAR $\gamma$ (ab8937, 1: 1000, Abcam), and anti-GAPDH polyclonal antibodies (ab9485, 1: 2500, Abcam) overnight $44^{\circ} \mathrm{C}$. The membrane was washed three times with PBS buffer containing $0.1 \%$ Tween, and the secondary antibody IgG-HRP (ab7090, 1: 2000, Abcam) was added and incubated for 1-2 hours. The PVDF membrane was exposed to DAB (3, 3'-diaminobenzidine, Sigma, USA) in a dark room and the relative expression of each protein was calculated by using ImageJ software (ImageJ software, USA). Semi-quantitative analysis was performed with GAPDH expression as the internal reference.

\section{Immunofluorescence}

The hASCs were inoculated into 12-well plates pre-coated with glass flakes after transfection for 72 h. After adipogenic differentiation induction for 7 days they were fixed in $4 \%$ paraformaldehyde. The cells were incubated with the blocking solution containing $10 \%$ sheep serum, $0.3 \%$ Triton X-100 and $0.1 \%$ sodium azide, then the primary antibodies anti-c/EBP $\alpha$ (ab32358, 1:50, Abcam) and anti-PPAR $\gamma$ (ab8937, $1 \mu \mathrm{g} / \mathrm{ml}, \mathrm{Abcam}$ ) were added and incubated at $4{ }^{\circ} \mathrm{C}$ overnight. A secondary antibody with Alexa Fluor 647 red fluorescence was added (ab150075, 1:200, Abcam) and incubated at $37^{\circ} \mathrm{C}$ for one hour (h). Hoechst was used to stain the nuclei and we observed the slides with a fluorescence microscope (Zeiss) after sealing.

\section{Double luciferase reporter assay}

The pGL3-WT plasmid was amplified and constructed by PCR using the fragment of the LPL 3'UTR containing the miR-27b binding site (positions 1287-1294). The pGL3-Mut plasmid was constructed by replacing the first six ribonucleotides of the complementary sequence in the LPL sequence. HEK293T cells were inoculated into 24-well plates with $4 \times 10^{3}$ cells / well and transfected according to the following four groups: miR-27b mimics+pGL3-WT (miR-WT), miR-27b mimics+pGL3-Mut (miR-Mut), control+pGL3-WT (NC-WT), control+pGL3-MuT (NC-Mut). After transfection for $24 \mathrm{~h}$, the firefly and bacilli luciferase reagents were added to detect the fluorescence activity of each group according to the double luciferase Reported Genetron Assay Kit (Beyotime Biotechnology, Shanghai, China).

\section{Statistical analysis}

GraphPad Prism (Version 6.0; La Jolla, CA, USA) was utilized to analyze the experimental data. The results are expressed as the mean \pm standard deviation. Two-sided $t$-tests and one-way ANOVA analysis were employed to perform statistical analysis and $P<0.05$ was considered statistically significant.

\section{Results}

miR-27b expression is decreased after adipogenic differentiation of hASCs

We used the GPL16770 platform to analyze two adipogenic differentiated samples and two non-adipogenic induction hASCs samples in GSE72429, which contains 16 samples. The screening criteria were fold change $>2$ and $P<0.05$, and 25 down-regulated miRNAs and 7 up-regulated miRNAs were identified. The expression level of miR-27b was reduced by 1.5 times after adipogenic differentiation (Fig. 1A-1B). The results of qRT-PCR showed 


\section{Cellular Physiology Cell Physiol Biochem 2018;47:545-555

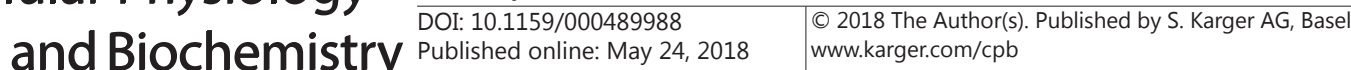

that the expression level of miR-27b was gradually down-regulated during adipogenesis induced differentiation and tended to stabilize after 14 days (Fig. 1C).

miR-27b inhibits adipogenic differentiation of hASCs

To verify the mechanism of miR-27bregulated adipogenic differentiation, we divided the hASCs into three groups: normal control (NC), miR-27b mimics and miR-27b inhibitor. After adipogenic induction differentiation for $0,1,7,14$ and 21 days, we detected the expression levels of adipogenic markers c/EBP $\alpha$ and PPAR $\gamma$. The results of qRT-PCR and western blot showed that 7 days after adipogenic differentiation, the expression levels of $\mathrm{c} / \mathrm{EBP} \alpha$ and PPAR $\gamma$ were significantly lower in the miR-27b mimics group and significantly higher in the miR-27b inhibitor group compared with the NC group. However, after 14 days of hASCs adipogenic differentiation, the increasing expression level of $\mathrm{c} / \mathrm{EBP} \alpha$ and PPAR $\gamma$ tended to stabilize in all three groups. Moreover, the results of immunofluorescence detected 14 days after adipogenic differentiation of hASCs showed that c/EBP $\alpha$ and PPAR $\gamma$ expression were increased in the miR-27b mimics group and decreased in the miR-27b inhibitor group (Fig. 2A-2C). In addition, after ad-

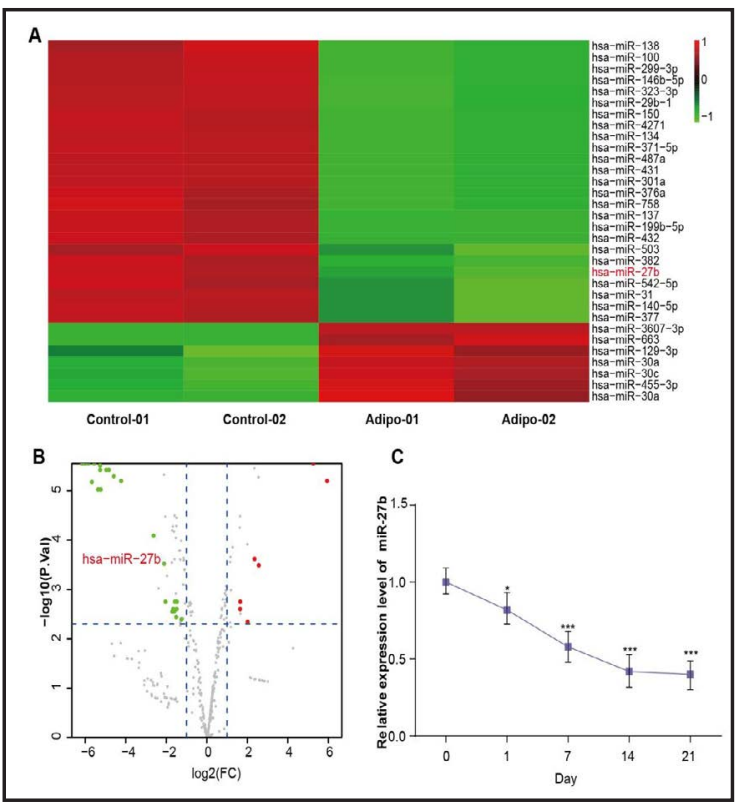

Fig. 1. MiR-27b expression is down-regulated during adipogenesis differentiation of hASCs. (A) Differential expression of micro-RNAs before and after adipogenic differentiation of hASCs are reflected in a heat map. (B) Volcano plot reveals the miRNAs differentially expressed before and after hASCs adipogenic differentiation and miR-27b expression are down-regulated after hASCs adipogenic differentiation. (C) The results of qRT-PCR show that miR-27b expression is gradually down-regulated during hASCs adipogenesis (from day 0 to day 21 ). ${ }^{*} \mathrm{P}<0.05,{ }^{* *} \mathrm{P}<0.01,{ }^{* * *} \mathrm{P}<0.001$, compared with day 0 .
Fig. 2. MiR-27b inhibits adipogenic differentiation of hASCs. (A-B) qRT-PCR and western blots show that $\mathrm{c} / \mathrm{EBP} \alpha$ and PPAR $\gamma$ expression are repressed by the miR-27b mimics but are promoted by the miR-27b inhibitor during adipogenic differentiation of hASCs (from day 0 to day 21 ), ${ }^{* * *} \mathrm{P}<0.001,{ }^{* * * *} \mathrm{P}<0.001$, compared with NC. (C) The expression levels of $\mathrm{c} / \mathrm{EBP} \alpha$ and PPAR $\gamma$ were repressed by the miR-27b mimics but promoted by the miR$27 \mathrm{~b}$ inhibitor after adipogenic differentiation of hASCs for 14 days shown by immunofluorescence, scale bar $=50 \mathrm{~mm}$. The positive cell ratios of $\mathrm{c} / \mathrm{EBP} \alpha$ and PPAR $\gamma$ were determined by the number of immune-positive cells to the number of nuclei stained with Hoechst. ${ }^{* *} \mathrm{P}<0.01,{ }^{* * *} \mathrm{P}<0.001,{ }^{* * * *} \mathrm{P}<0.001$, compared with NC.

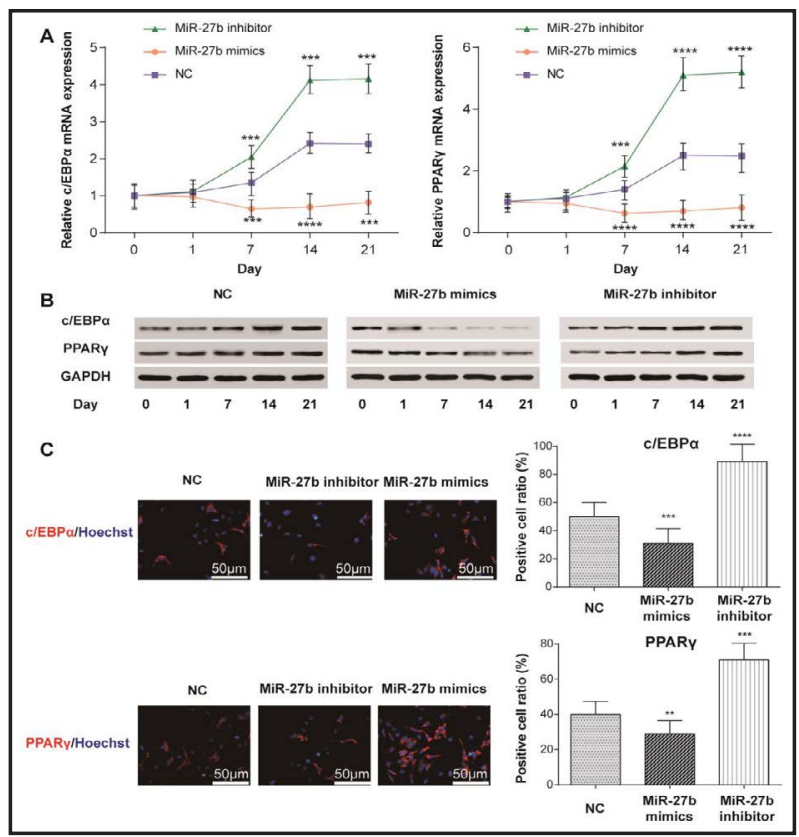


ipogenic induction differentiation for 7 and 14 days, we detected fat droplet accumulation by the oil red 0 staining assay. The results showed that the fat droplet accumulation after adipogenic differentiation of hASCs at both 7 and 14 days were reduced in the miR$27 \mathrm{~b}$ mimics group. Nevertheless, the accumulation of fat droplets was increased in the miR-27b inhibitor group (Fig. 3A-3B).

LPL is highly expressed after adipogenic differentiation and is regulated by $\mathrm{miR}-27 \mathrm{~b}$

The GPL16686 platform was used to analyze differentially expressed mRNA during adipogenic differentiation of hASCs in GSE77532. The hASCs underwent adipocyte differentiation for 1, 7, 14 and 21 days, representing the early to late stage process of adipogenesis. First, multivariate analysis of variance showed that there were no significantly differentially expressed mRNAs during routine culture of hASCs treated without adipogenesis induction. However, several mRNAs were highly expressed on the $1^{\text {st }}$ day of adipogenic differentiation relative to the $7^{\text {th }}, 14^{\text {th }}$ and $21^{\text {st }}$ day in hASCs. Other mRNAs expression presented the opposite pattern. Meanwhile, we found that mRNAs expression gradually increased or decreased during adipogenesis induction and reached an extreme on the $14^{\text {th }}$ day. The mRNA expression level then tended to steady or recover. In short, the mRNA expression levels showed no significant changes under general culture conditions but could be regulated by adipogenic differentiation in hASCs.

Using ANOVA analysis with the screening criterion of $P<0.001,6$ down-regulated mRNAs and 35 up-regulated mRNAs were identified during adipogenic differentiation of hASCs for 1, 7, 14 and 21 days (Fig. 4A). The microRNA $L P L$ was up-regulated during adipogenesis differentiation and selected for further studies. The results of qRT-PCR revealed that the expression of $L P L$ was significantly increased from the $7^{\text {th }}$ day of adipogenic differentiation and tended to stabilize on the $21^{\text {st }}$ day of adipogenic differentiation (Fig. 4B) in hASCs.

To explore the relationship between miR-27b and $L P L$, we detected the expression level of $L P L$ among three different groups: NC, the miR-27b mimics group and the miR-27b inhibitor group. The results of qRT-PCR and western blot consistently showed that $L P L$ was expressed at low levels during adipogenic differentiation in the miR-27b mimics group but was highly expressed in the miR-27b inhibitor group compared with the NC group, $P<0.01$. Similarly, $L P L$ expression at day 21 of adipogenesis showed a reversed tendency in each group (Fig. 4C-4D). In summary, $L P L$ was highly expressed during adipogenic differentiation and reversibly regulated by miR-27b.

MiR-27b targeted LPL

The TargetScan database was used to search for the mRNA containing the complementary sequence of miR-27b. As shown in Fig. 5A, there was a potential binding site for miR- 
Fig. 4. LPL is gradually up-regulated during adipogenesis differentiation of hASCs. (A) Heat map showing the differentially expressed mRNAs during adipogenic differentiation of hASCs. (B) The results of qRT-PCR revealed the LPL expression level was up-regulated during adipogenesis differentiation of hASCs (from day 0 to day 21). ${ }^{* *} \mathrm{P}<0.01, \quad{ }^{* * *} \mathrm{P}<0.001, \quad{ }^{* * * *} \mathrm{P}<0.001$, compared with the first day. (C) The results of qRT-PCR revealed the expression of LPL was decreased in the miR-27b mimics group and increased in the miR-27b inhibitor group compared with $\mathrm{NC}$. ${ }^{* *} \mathrm{P}<0.01,{ }^{* * *} \mathrm{P}<0.001$, compared with NC. (D) Western blot analysis and quantification demonstrating that the LPL protein expression level was repressed by miR-27b but promoted by a miR-27b inhibitor during adipogenic differentiation of hASCs (from day 0 to day 21). ${ }^{* *} \mathrm{P}<0.01,{ }^{* * *} \mathrm{P}<0.001$, compared with NC.

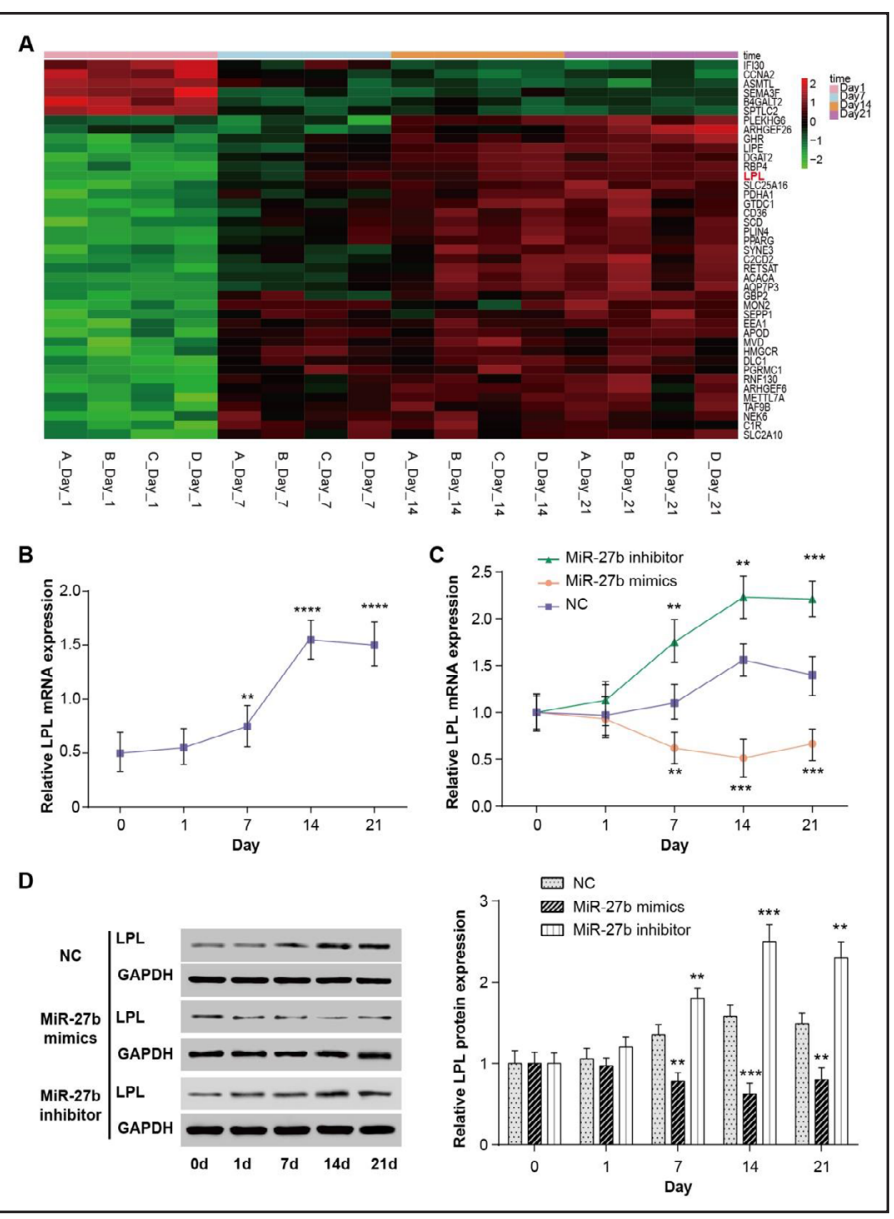

$27 \mathrm{~b}$ in the 3'UTR region of $L P L$, which indicated that $L P L$ might be targeted by miR-27b. A dual luciferase reporter assay was used to examine the direct targeting relationship between $L P L$ and miR-27b. The results indicated that the relative luciferase activity of the group co-transfected with miR-27b mimics+pGL3-WT displayed a significant decline compared with the groups co-transfected with miR-27b mimics+pGL3Mut, control mimics+pGL3-WT and control mimics+pGL3-Mut (Fig. 5B), confirming the targeting relationship between miR$27 \mathrm{~b}$ and $L P L$.

LPL promotes hASCs adipogenic differentiation

To discover the mechanism

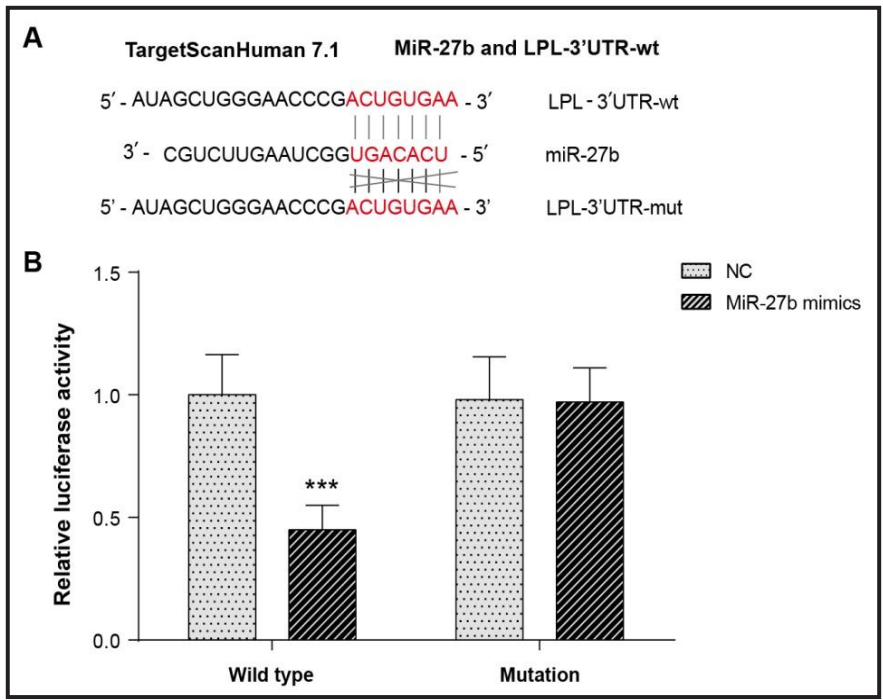

Fig. 5. Relationship between miR-27b and LPL. (A) TargetScan Database showing the binding site between the miR-27b and the 3'UTR region of LPL. (B) Dual luciferase reporter assay suggests miR-27b regulates adipogenic differentiation of hASCs through targeting LPL. ${ }^{* * *} \mathrm{P}<0.001$, compared with NC. whereby $L P L$ regulates hASCs 
adipogenic differentiation, four transfection groups were used to study adipogenic differentiation: NC, pcDNA3.1-LPL, LPL shRNA and miR-27b mimics+pcDNA3.1LPL. The expression of the fat-formationrelated biomarkers $\mathrm{c} / \mathrm{EBP} \alpha$ and PPAR $\gamma$ and the fat droplet accumulation level were detected after adipogenic differentiation at 0 day, 1 day, 7 days, 14 days and 21 days. The results of qRT-PCR and western blot indicated that the expression levels of c/EBP $\alpha$ and PPAR $\gamma$ in the pcDNA3.1-LPL group were significantly higher than that in the NC group after adipogenic differentiation for 7 days, while it was reduced in the LPL shRNA group. However, the changing trend during adipogenesis recovered on day 21 of adipogenic differentiation. Meanwhile, the miR-27b mimics+pcDNA3.1-LPL group displayed the same tendency as the NC group (Fig. 6A-6B). In addition, the results of immunofluorescence testing for $\mathrm{c} / \mathrm{EBP} \alpha$ and PPAR $\gamma$ expression on the $14^{\text {th }}$ day of hASCs adipogenic differentiation corresponded with the results of the qRTPCR and western blot (Fig. 6C-6D). Oil red 0 staining was utilized to detect fat droplet accumulation and the results revealed that fat droplet accumulation was increased in the pcDNA3.1-LPL group after adipogenic differentiation for 7 and 14 days compared with that in the NC group. Fat droplet accumulation in the LPL shRNA group presented a significant decline relative to that in the NC group, while fat droplet accumulation in the miR-27b mimics+pcDNA3.1-LPL group was similar to that in the NC group (Fig. 7A-7B). These findings indicate that $L P L$ promoted hASCs adipogenic differentiation and is regulated by miR-27b.

\section{Discussion}

In this study, we first observed that

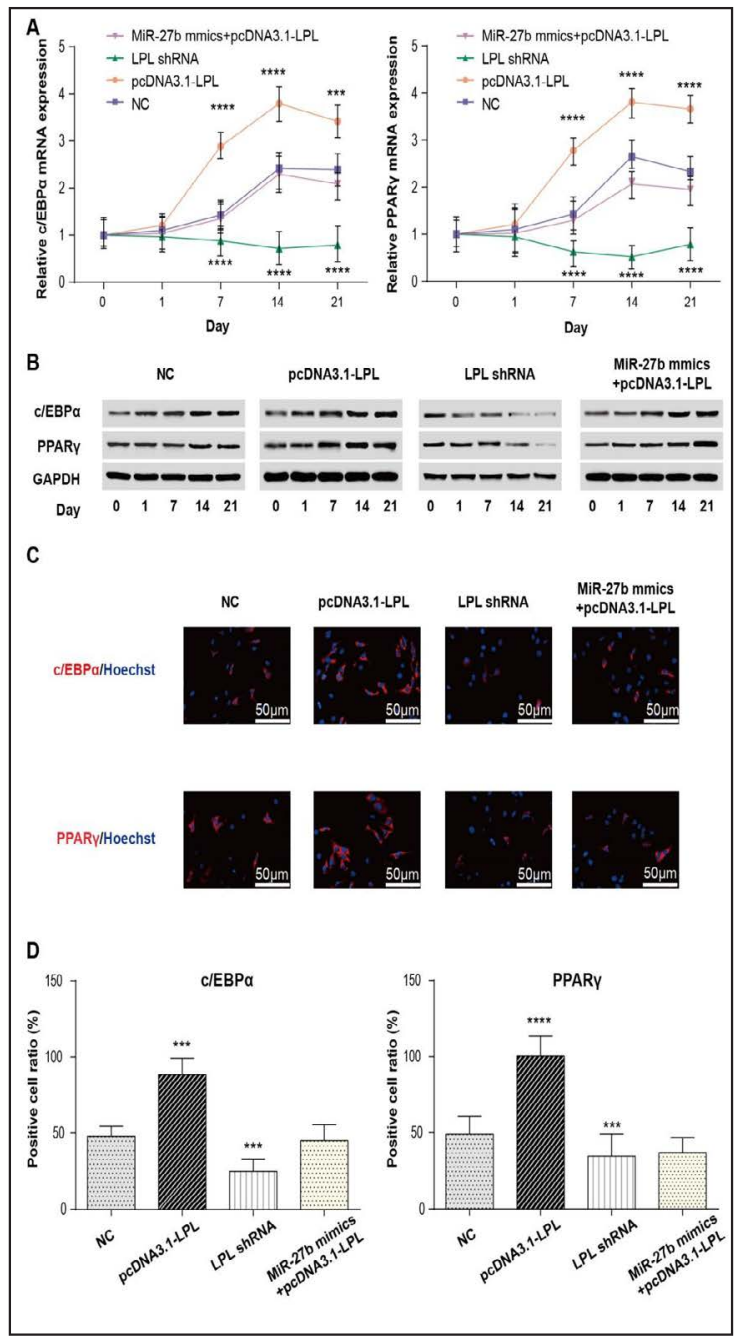

Fig. 6. LPL promotes adipogenic differentiation of hASCs. (A-B) qRT-PCR and western blot show that c/ $\mathrm{EBP} \alpha$ and PPAR $\gamma$ expression are promoted by overexpressed LPL but repressed by LPL shRNA during adipogenic differentiation of hASCs (from day 0 to day 21). ${ }^{* * *} \mathrm{P}<0.001,{ }^{* * * *} \mathrm{P}<0.001$, compared with NC. (C-D) The expression levels of $c / E B P \alpha$ and PPAR $\gamma$ are promoted by LPL but repressed by LPL shRNA after adipogenic differentiation of hASCs for 14 days as shown by immunofluorescence. Scale bar $=50 \mathrm{~mm}$. The positive cell ratios of $\mathrm{c} / \mathrm{EBP} \alpha$ and PPAR $\gamma$ were determined by the ratio of the number of immune-positive cells to the number of nuclei stained with Hoechst. ${ }^{* * *} \mathrm{P}<0.001$, $* * * * \mathrm{P}<0.001$, compared with NC. miR-27b was expressed at low levels during hASCs adipogenic differentiation, and we eventually proved that miR-27b could inhibit hASCs adipogenic differentiation. The differential expression of adipogenic markers c/EBP $\alpha$ and PPAR $\gamma$ among the three groups demonstrated that the adipogenic differentiation was regulated by miR-27b and had a negative connection between them [14, 22-24]. Additionally, the oil red 0 staining assay detected fat droplet accumulation in hASCs, further proving that over-expression of miR-27b could inhibit hASCs adipogenic differentiation. 


\section{Cellular Physiology \begin{tabular}{l|l} 
and Biochemistry Published online: May 24, 2018 & $\begin{array}{l}\text { C } 2018 \text { The Author(s). Published by S. Karger AG, Basel } \\
\text { www.karger.com/cpb }\end{array}$
\end{tabular} \\ Hu et al.: Up-Regulation of miR-27b Inhibits Adipogenic Differentiation of hASCs}

Fig. 7. LPL promoted adipogenic differentiation of hASCs by oil red 0 staining. (A-B) The fat droplet accumulation was increased in the pcDNA3.1-LPL group and decreased in the LPL shRNA group after adipogenic differentiation of hASCs cells for 7 and 14 days as detected by oil red 0 staining and the histogram shows quantification of oil red 0 staining by spectrophotometry. Bar $=50 \mathrm{~mm}, * \mathrm{P}<0.05,{ }^{* * *} \mathrm{P}<0.001$, $* * * * \mathrm{P}<0.001$, compared with NC.

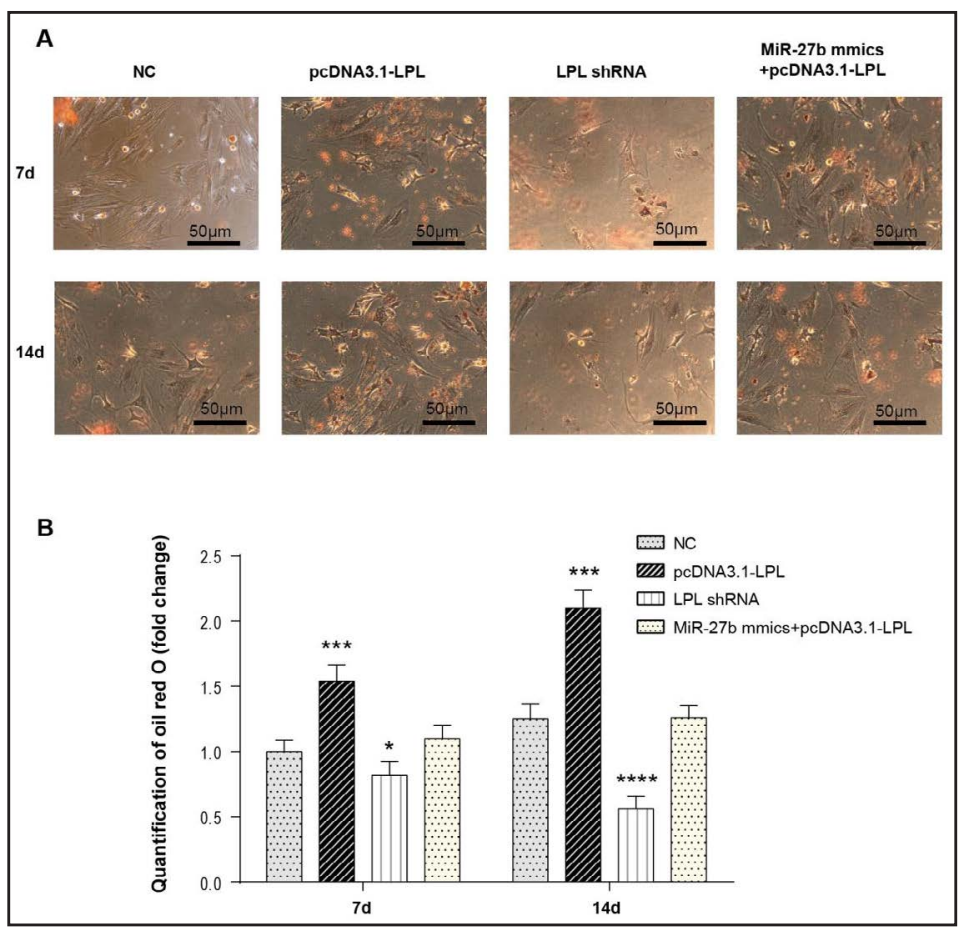

The in vivo differentiation process of hASCs is very complicated and involves various internal signals such as FGF and Erk pathways. Furthermore, chemicals or small molecules including cytokines are major factors in the induction of cell differentiation [25-28]. Francisco et al. found that human preadipocytes and ASCs were excellent models for studying adipogenesis and obesity-related metabolic alterations and were appropriate for revealing adipocyte renewal, development and depot-specific differences [4]. Therefore, we focused on uncovering the molecular regulation mechanism as to how miRNAs regulate adipogenic differentiation of hASCs.

miRNAs are 18-24 nucleotides, single-stranded, non-coding RNA. They have been proven to be involved in various tissues and biological functions [29]. For example, miR141 and miR-200a have been reported to regulate osteoblast differentiation by targeting the BMP-2 signaling pathway [30]. MiR-346 regulates hBMSCs osteoblast differentiation by targeting GSK-3 $\beta$ and activating the Wnt/ $\beta$-catenin pathway [31]. Moreover, miR-27b has been verified to participate in lipid metabolism and adipogenesis $[32,33]$, which is important for revealing these mechanisms and finding treatments for obesity, diabetes mellitus, and other obesity related metabolic diseases. In our study, we identified that miR-27b expression was significantly lower during adipogenic differentiation. Subsequently, reduced expression of miR-27b promoted the adipogenic differentiation of hASCs.

The findings from miRNA investigations during adipogenesis suggest a potential role of miRNA mimics/inhibitors in treating bone diseases, metabolic disorders, and obesity [5, 34]. The role of the lipoprotein lipase $(L P L)$ gene in adipogenesis has been previously studied and expression of $L P L$ messenger RNA was observed in the early stages of adipogenesis and it reached a stable level in mature adipocytes, which indicates $L P L$ is one of the critical factors in the process of adipogenic differentiation [35]. We also observed that LPL was highly expressed during adipogenic differentiation of hASCs and proved that $L P L$ was negatively regulated by miR-27b. In addition, we certified that LPL promoted hASCs adipogenic differentiation. Meanwhile, we confirmed the target regulation relationship between miR$27 \mathrm{~b}$ and $L P L$, and their individual functions in hASCs adipogenic differentiation.

One of the limitations in this study was that the analyzed samples used to detect differentially expression miRNA were few in number. Nonetheless, we report that miR$27 \mathrm{~b}$ overexpression inhibited adipogenic differentiation, to a certain extent, ensuring miR- 


\section{Cellular Physiology Cell Physiol Biochem 2018;47:545-555 \begin{tabular}{l|l} 
and Biochemistry Published online: May 24, 2018 & $\begin{array}{l}\text { (c) } 2018 \text { The Author(s). Published by S. Karger AG, Basel } \\
\text { www.karger.com/cpb }\end{array}$
\end{tabular}}

Hu et al.: Up-Regulation of miR-27b Inhibits Adipogenic Differentiation of hASCs

$27 \mathrm{~b}$ is down-up-regulated during adipogenic differentiation. Additionally, the detailed action mechanisms of the miR-27b/c/EBP $\alpha / P P A R \gamma$ axis and the $L P L / c / E B P \alpha / P P A R \gamma$ axis regulating adipogenic differentiation of hASCs cells are still elusive and remained to be further investigated. However, the molecular mechanism research performed in this study was conducive to explore adipogenesis and discover obesity-related metabolic alterations.

In summary, we identified a targetable regulation relationship between miR-27b and $L P L$ and their individual functions in hASCs adipogenic differentiation. These results may provide new therapeutic targets, which will facilitate our development of therapies for these diseases.

\section{Conclusion}

In conclusion, we showed that miR-27b expression decreased and $L P L$ expression increased during adipogenic differentiation of hASCs and abnormal expression of miR-27b and $L P L$ played a significant role in regulating adipogenic differentiation.

\section{Disclosure Statement}

No conflict of interests exists.

\section{References}

1 Cinti S: The adipose organ at a glance. Dis Model Mech 2012;5:588-594.

- Cinti S: Between brown and white: Novel aspects of adipocyte differentiation. Ann Med 2011;43:104-115.

-3 Minteer D, Marra KG, Rubin JP: Adipose-derived mesenchymal stem cells: Biology and potential applications. Adv Biochem Eng Biotechnol 2013;129:59-71.

-4 Ruiz-Ojeda FJ, Ruperez AI, Gomez-Llorente C, Gil A, Aguilera CM: Cell models and their application for studying adipogenic differentiation in relation to obesity: A review. International journal of molecular sciences 2016;17

5 Hamam D, Ali D, Kassem M, Aldahmash A, Alajez NM: Micrornas as regulators of adipogenic differentiation of mesenchymal stem cells. Stem Cells Dev 2015;24:417-425.

6 Rosen ED, Spiegelman BM: Molecular regulation of adipogenesis. Annu Rev Cell Dev Biol 2000;16:145-171.

7 Liu B, Chen W, Cao G, Dong Z, Xu J, Luo T, Zhang S: Microrna-27b inhibits cell proliferation in oral squamous cell carcinoma by targeting fzd7 and wnt signaling pathway. Arch Oral Biol 2017;83:92-96.

-8 He H, Jazdzewski K, Li W, Liyanarachchi S, Nagy R, Volinia S, Calin GA, Liu CG, Franssila K, Suster S, Kloos RT, Croce CM, de la Chapelle A: The role of microrna genes in papillary thyroid carcinoma. Proc Natl Acad Sci U S A 2005;102:19075-19080.

-9 Karp X, Ambros V: Developmental biology. Encountering micrornas in cell fate signaling. Science 2005;310:1288-1289.

10 Ambros V: The functions of animal micrornas. Nature 2004;431:350-355.

-11 Sun J, Wang Y, Li Y, Zhao G: Downregulation of ppargamma by mir-548d-5p suppresses the adipogenic differentiation of human bone marrow mesenchymal stem cells and enhances their osteogenic potential. J Transl Med 2014;12:168.

12 Zhang M, Wu JF, Chen WJ, Tang SL, Mo ZC, Tang YY, Li Y, Wang JL, Liu XY, Peng J, Chen K, He PP, Lv YC, Ouyang XP, Yao F, Tang DP, Cayabyab FS, Zhang DW, Zheng XL, Tian GP, Tang CK: Microrna-27a/b regulates cellular cholesterol efflux, influx and esterification/hydrolysis in thp-1 macrophages. Atherosclerosis 2014;234:5464.

13 Chen SZ, Xu X, Ning LF, Jiang WY, Xing C, Tang QQ, Huang HY: Mir-27 impairs the adipogenic lineage commitment via targeting lysyl oxidase. Obesity (Silver Spring) 2015;23:2445-2453.

14 Kim SY, Kim AY, Lee HW, Son YH, Lee GY, Lee JW, Lee YS, Kim JB: Mir-27a is a negative regulator of adipocyte differentiation via suppressing ppargamma expression. Biochem Biophys Res Comm 2010;392:323-328. 


\section{Cellular Physiology Cell Physiol Biochem 2018;47:545-555 \begin{tabular}{l|l} 
and Biochemistry Published online: May 24, 2018 & $\begin{array}{l}\text { (c) } 2018 \text { The Author(s). Published by S. Karger AG, Basel } \\
\text { www.karger.com/cpb }\end{array}$
\end{tabular} \\ Hu et al.: Up-Regulation of miR-27b Inhibits Adipogenic Differentiation of hASCs}

15 Jia YJ, Liu ZB, Wang WG, Sun CB, Wei P, Yang YL, You MJ, Yu BH, Li XQ, Zhou XY: Hdac6 regulates microrna$27 \mathrm{~b}$ that suppresses proliferation, promotes apoptosis and target met in diffuse large b-cell lymphoma. Leukemia 2017

16 Tao J, Zhi X, Zhang X, Fu M, Huang H, Fan Y, Guan W, Zou C: Mir-27b-3p suppresses cell proliferation through targeting receptor tyrosine kinase like orphan receptor 1 in gastric cancer. J Exp Clin Cancer Res 2015;34:139.

17 Zhang G, Tian X, Li Y, Wang Z, Li X, Zhu C: Mir-27b and mir-34a enhance docetaxel sensitivity of prostate cancer cells through inhibiting epithelial-to-mesenchymal transition by targeting zeb1. Biomed Pharmacother 2018;97:736-744.

-18 Sha H, Sun S, Francisco AB, Ehrhardt N, Xue Z, Liu L, Lawrence P, Mattijssen F, Guber RD, Panhwar MS, Brenna JT, Shi H, Xue B, Kersten S, Bensadoun A, Peterfy M, Long Q, Qi L: The er-associated degradation adaptor protein sel11 regulates lpl secretion and lipid metabolism. Cell Metab 2014;20:458-470.

19 Takagi A, Ikeda Y: [genetic diagnosis on hypertriglyceridemia-analysis for lpl gene mutations]. Nihon Rinsho 2013;71:1569-1576.

20 Glaser DS, Yost TJ, Eckel RH: Preheparin lipoprotein lipolytic activities: Relationship to plasma lipoproteins and postheparin lipolytic activities. J Lipid Res 1992;33:209-214.

21 Estes BT, Diekman BO, Gimble JM, Guilak F: Isolation of adipose-derived stem cells and their induction to a chondrogenic phenotype. Nat Protoc 2010;5:1294-1311.

22 Zhu Y, Zhang X, Ding X, Wang H, Chen X, Zhao H, Jia Y, Liu S, Liu Y: Mir-27 inhibits adipocyte differentiation via suppressing creb expression. Acta Biochim Biophys Sin (Shanghai) 2014;46:590-596.

23 Kang T, Lu W, Xu W, Anderson L, Bacanamwo M, Thompson W, Chen YE, Liu D: Microrna-27 (mir-27) targets prohibitin and impairs adipocyte differentiation and mitochondrial function in human adipose-derived stem cells. J Biol Chem 2013;288:34394-34402.

-24 Lin Q, Gao Z, Alarcon RM, Ye J, Yun Z: A role of mir-27 in the regulation of adipogenesis. FEBS J 2009;276:2348-2358.

-25 Schmole AC, Hubner R, Beller M, Rolfs A, Frech MJ: Small molecules in stem cell research. Curr Pharm Biotechnol 2013;14:36-45.

26 Kunisada Y, Tsubooka-Yamazoe N, Shoji M, Hosoya M: Small molecules induce efficient differentiation into insulin-producing cells from human induced pluripotent stem cells. Stem Cell Res 2012;8:274-284.

27 Miraoui H, Oudina K, Petite H, Tanimoto Y, Moriyama K, Marie PJ: Fibroblast growth factor receptor 2 promotes osteogenic differentiation in mesenchymal cells via erk1/2 and protein kinase c signaling. J Biol Chem 2009;284:4897-4904.

28 Jaiswal RK, Jaiswal N, Bruder SP, Mbalaviele G, Marshak DR, Pittenger MF: Adult human mesenchymal stem cell differentiation to the osteogenic or adipogenic lineage is regulated by mitogen-activated protein kinase. J Biol Chem 2000;275:9645-9652.

29 Gu C, Xu Y, Zhang S, Guan H, Song S, Wang X, Wang Y, Li Y, Zhao G: Mir-27a attenuates adipogenesis and promotes osteogenesis in steroid-induced rat bmscs by targeting ppargamma and grem1. Sci Rep 2016;6:38491.

-30 Itoh T, Nozawa Y, Akao Y: Microrna-141 and -200a are involved in bone morphogenetic protein-2-induced mouse pre-osteoblast differentiation by targeting distal-less homeobox 5. J Biol Chem 2009;284:1927219279.

-31 Wang Q, Cai J, Cai XH, Chen L: Mir-346 regulates osteogenic differentiation of human bone marrow-derived mesenchymal stem cells by targeting the wnt/beta-catenin pathway. PloS one 2013;8:e72266.

-32 Shi C, Huang F, Gu X, Zhang M, Wen J, Wang X, You L, Cui X, Ji C, Guo X: Adipogenic mirna and meta-signature mirnas involved in human adipocyte differentiation and obesity. Oncotarget 2016;7:40830-40845.

-33 Huang J, Zhao L, Xing L, Chen D: Microrna-204 regulates runx2 protein expression and mesenchymal progenitor cell differentiation. Stem Cells 2010;28:357-364.

-34 Eulalio A, Mano M, Dal Ferro M, Zentilin L, Sinagra G, Zacchigna S, Giacca M: Functional screening identifies mirnas inducing cardiac regeneration. Nature 2012;492:376-381.

-35 Kim D, Kim J, Hyun H, Kim K, Roh S: A nanoscale ridge/groove pattern arrayed surface enhances adipogenic differentiation of human supernumerary tooth-derived dental pulp stem cells in vitro. Arch Oral Biol 2014;59:765-774. 